\title{
Simulation Study of the Volatility of Order Sizes and Their Impact on the Stability of a Simple Manufacturing Environment
}

\author{
Peter Nielsen ${ }^{1, *}$ and Grzegorz Bocewicz ${ }^{2}$ \\ ${ }^{1}$ Department of Mechanical and Manufacturing Engineering, \\ Aalborg University, Fibigerstraede 16, DK 9220 Aalborg Oest, Denmark \\ peter@m-tech.aau.dk \\ ${ }^{2}$ Koszalin University of Technology, \\ Dept. of Computer Science and Management, Koszalin, Poland \\ bocewicz@ie.tu.koszalin.pl
}

\begin{abstract}
Customer ordering behaviorin the form of size and timing of orders are critical for manufacturing systems such as make-to-order or assemble-toorder systems. To be competitive a Make-to-order manufacture must be flexible, both with regards to volume and product mix. A particularly critical parameter in determining the flexibility needs in the case of responsive manufacturing environments is the distribution of order sizes. In this paper several methods for describing the volatility of order sizes are presented. A discrete event simulation of a single server batch manufacturing system is subsequently conducted using various distributions of order sizes. The aim is to investigate 1) which measures for order size volatility best relates to the volatility of the output rate from the manufacturing system 2) how does the output stability from the system deteriorate as order sizes become more volatile.
\end{abstract}

Keywords: Simulation study, order sizes, discrete event.

\section{Introduction}

The performance of planning approaches within Manufacturing Planning and Control to a large extend depends on the planning foundation [6]. This foundation is for the purpose of this paper be limited to a simple model consisting of external demand / supply conditions, internal structure (planning approach and customer order decoupling points, Bill-of-Material/Bill-of-Resources structure and Product family structure). The internal structure should match the external conditions and enable an effective and efficient transformation of input from suppliers (materials and resources) to matching customer requirements [11]. For simplicity's sake this paper will focus on the orders arriving to the systems and more specifically to the size of orders rather than the timing of their arrival. The timing of orders rightfully falls in

\footnotetext{
* Corresponding author.
} 
the domain of demand forecasting modeling, where this paper focuses on the response to the order sizes themselves. The considered system will be a simple single server system responding directly to customer orders. This system could correspond to a simple make-to-order manufacturing system. This paper has three main contributions. First, a method for evaluating the variation of order sizes is discussed. Second, a discrete event simulation study of a single server system is conducted to investigate the impact of order size volatility on a make-to-order system. Third, various measures for describing order size volatility are compared to the stability of the output from the manufacturing system to identify which order size volatility measure best predicts the volatility of the output. The remainder of the paper is structured as follows. First, a brief literature review is presented. Second, a new method for evaluating order size volatility is presented. Following this a simulation study of the impact of order size volatility on the output is presented. This is followed by a section discussing the experimental results before finally conclusions and further research is presented.

\section{$2 \quad$ Literature Review}

Orders form the foundation for demand. However, they typically act in an unpredictable manner [6], and are as a consequence typically aggregated to give stability and to serve as a planning input [10]. Wijngaard [12] identify four normal dimensions for aggregation: Types of products, production stages, capacities and time. Of these the types of products and time are the two typical used dimensions when aggregating demand information, as seen in e.g. Vollmann et al. [11]. Especially the time dimension is often used so that orders are aggregated over time in a way that ensures that the aggregated demand information can be translated into allocations in the internal manufacturing system structure [11]. The further up-stream the customer order decoupling point is placed, the more a company will be reacting directly to the customer ordering information as structured in the customer order [1], [7]. This also means that the planning approach is adapted according to the customer ordering decoupling point [8] and that impact of variation in order sizes will depend on the context. The consequence is that while it may be possible to allocate resources, materials and balance manufacturing lines pre-ante based on aggregate forecasts of demand translated into demand rates for individual products, the system it-self will in fact be reacting to a customer ordering behavior that may differ significantly from the assumptions. So while aggregate demand may be stable [13] and comply with standard assumptions such as stationary demand rates [3] the output may in fact be unstable or dependent. Because of this, it is relevant to investigate the behavior of customer demand in the form of the order sizes and the impact on a simple manufacturing system. This also has a number of further implications for e.g. line balancing that is highly sensitive to the distribution of orders [4]. In the case of line balancing it will be of significant value to have not just a clear picture of overall volumes and mix of orders, but also have a clear idea of the size of orders per product received. The more volatile the order sizes, the more difficult line balancing becomes [4]. For these reasons it is critical to have a clear picture of the volatility of order sizes faced by the company and how the manufacturing system reacts to these. This paper focuses on the second point. 


\section{$3 \quad$ Method for Evaluating Order Size Volatility}

An often used term to describe the volatility of a given distribution is the Coefficient of Variance (CV), the standard deviation of the observed values, divided by the mean of the observed values. $\mathrm{CV}$ can be adapted to describe the volatility of order sizes by $\boldsymbol{C} \boldsymbol{V}_{\boldsymbol{Q}}=\frac{\sigma_{Q}}{\mu_{Q}}$, where $\sigma_{\mathrm{Q}}$ is the standard deviation of order sizes and $\mu_{\mathrm{Q}}$ is the mean order size. This term is particular suitable if the observations are i.i.d. from e.g. the normal distribution. However, since the likelihood of normality is limited we use another term to establish the volatility of order sizes [5]. The measure of variation is established as the following. First, sort the orders for the period by size. Second, calculate the accumulated demand for the sorted set of orders, i.e. the increase to total demand by adding the next order. Third, calculate the relative amount of total demand for all percentiles of orders. In the ideal situation of constant order sizes one order will proportionally increase the accumulated demand as much as any other orders. This means that e.g. $25 \%$ of the orders should amount to precisely $25 \%$ of the total demand. The higher the $\mathrm{CV}$, the more one would expect the percentiles to deviate. Figure 1 illustrates the proposed measure of order size stability, with the three percentiles $90 \%, 95 \%$ and $98 \%$ illustrated on the bottom graph with respectively a green, blue and red line.
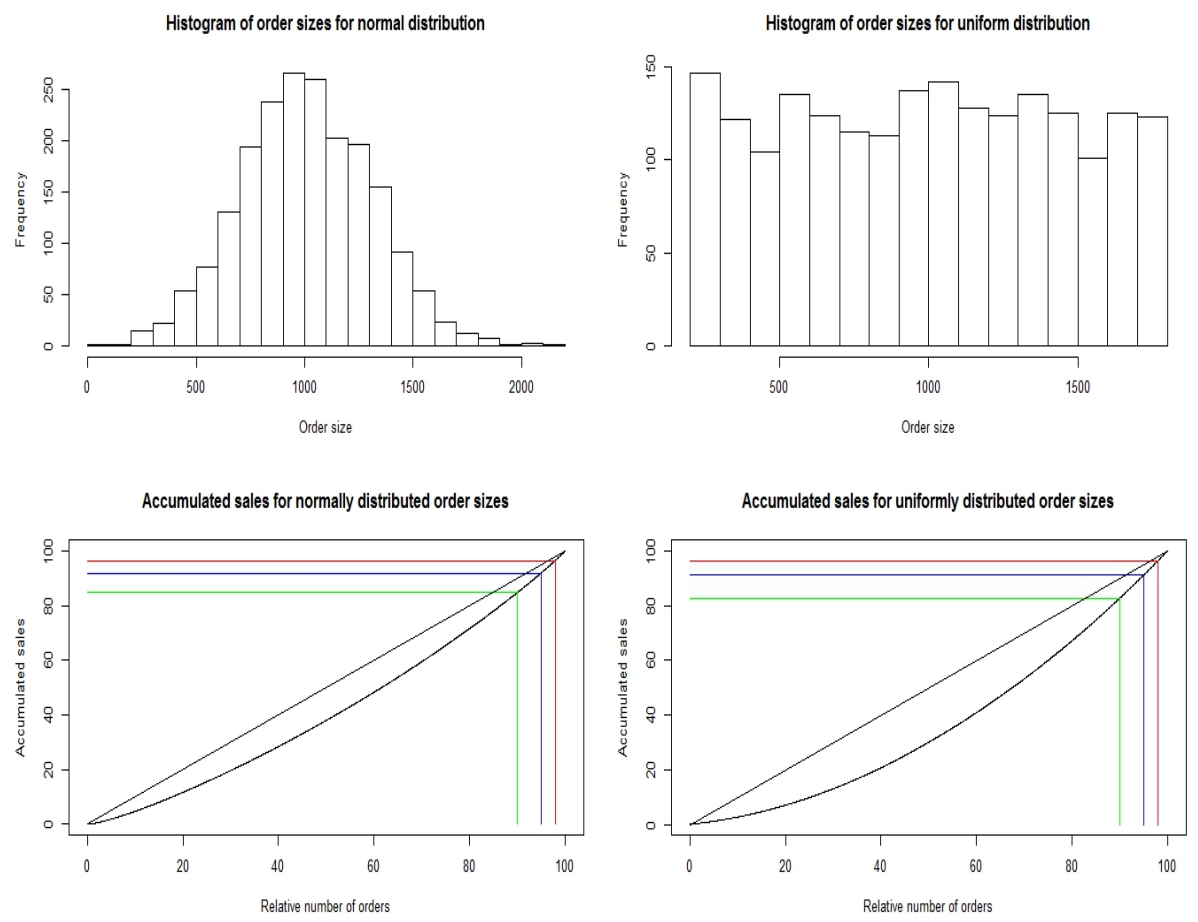

Fig. 1. Top: Histogram of order sizes from a normal (left) and uniform (right) distribution. Bottom: The corresponding accumulated demand profiles. 
The order size distributions shown in figure 1 are 2,000 observations from a normal distribution, with a mean of 1000 and standard deviation of $300(\mathrm{CV}=0.3)$ and a uniform distribution with minimum 200 and a maximum of 1800 (approx. $\mathrm{CV}=0.46$ ). The bottom graphs in figure 2 illustrate the concept of variation of order size, while the histograms illustrate the distributions. The curved graphs are the accumulated demand with orders sorted by order size. The lower the corresponding value is for a given percentile, the further from the assumption of constant order size is from actual distribution and the higher the $\mathrm{CV}$ will be. As such the percentiles become substitute measures describing the relative skewness of a given distribution. The more the distribution deviates from symmetry the lower values will be achieved for a given percentile.

\section{Simulation of Impact of Volatility on Planning Environment Stability}

The experimental setup has three aspects, the various data sets of order sizes used, the simulated manufacturing system and the measures used to describe the input and output. The considered manufacturing system consists of a single server processing orders from an infinite queue taking orders FIFO. The server processes the orders as batches and the orders exit the system as a batch when all the order has been processed. The server has the following properties:

- Process times dependent on order size, and stem from a i.i.d. normal distribution, with a situational mean of $Q_{j} * \operatorname{Pr} T$ and standard deviation $\sqrt{Q_{j}} * \sigma_{\operatorname{PrT}}$, where $\mathrm{Q}_{\mathrm{j}}$ is the order size of order $j$, and $\operatorname{PrT}$ is the average processing time per unit, and $\sigma_{\mathrm{PrT}}$ is the standard deviation per processed unit. Processing times are i.i.d.

- Setup time is constant and independent of order size and set to $\frac{\mu_{Q * \operatorname{Pr} T}}{2}$.

- Orders are batch processed and no units of the order are released until the whole order is completed.

- $\quad \operatorname{PrT}$ is in the simulation set to $1 \mathrm{~min}$. /unit and $\sigma_{\operatorname{PrT}}$ is set to $0.3^{*} \operatorname{PrT}$.

Order sizes are drawn from the following distributions (and rounded to integers):

- $\quad$ sets from normal distributions with CV's $0.05,0.10,0.15,0.20,0.25,0.30$, (labeled respectively norm_set_1:6)

- $\quad$ sets from uniform distributions with limits 18:22, 15:25, 12:28, 9:31, 6:34 and 3:37 (labeled respectively uni_set_1:6)

- 1 set from a an exponential distribution (exp_set)

- 1 set with constant order size (cst_set)

All 14 order size distributions have a mean $\left(\mu_{\mathrm{Q}}\right)$ of 20 units.

To evaluate the stability of the order sizes for the various distributions the order size distribution scheme proposed in the previous section is used in combination with 
the $\mathrm{CV}_{\mathrm{Q}}$. The order distribution scheme allows for the calculation of any number of percentiles of order sizes and their contribution to the total processed demand. In this case the $30 \%, 40 \%, 50 \%, 60 \%, 70 \%, 80 \%, 90 \%, 95 \%, 98 \%$ and $99 \%$-percentiles were chosen. To evaluate the stability of the manufacturing system under investigation the following measures are proposed:

$C V_{V, i}=\frac{\sigma_{V, i}}{\mu_{V, i}}$, where $\sigma_{\mathrm{V}, \mathrm{i}}$ is the standard deviation of total demand processed (i.e. volume in units outputted) in a time interval of $i$ and $\mu_{\mathrm{V}, \mathrm{i}}$ is the mean demand processed in time interval $i$.

$C V_{N, i}=\frac{\sigma_{N, i}}{\mu_{N, i}}$, where $\sigma_{\mathrm{N}, \mathrm{i}}$ is the standard deviation of the number of orders processed in a time interval of length $i$ and $\mu_{\mathrm{N}, \mathrm{i}}$ is the mean number of orders processed in a time interval of length $i$.

\section{$5 \quad$ Experimental Results}

For data analysis and data generation the statistical software package R [9] is used, while Enterprise Dynamics $\bigodot$ is used for the discrete event simulation. A simulated manufacturing run was conducted for 200 hours of manufacturing time. The results of the output variation and order size characteristics are shown below in table 1 and figure 3 and the order size distributions' variation are shown in figure 2 .

\section{Order size distribution for all data sets}

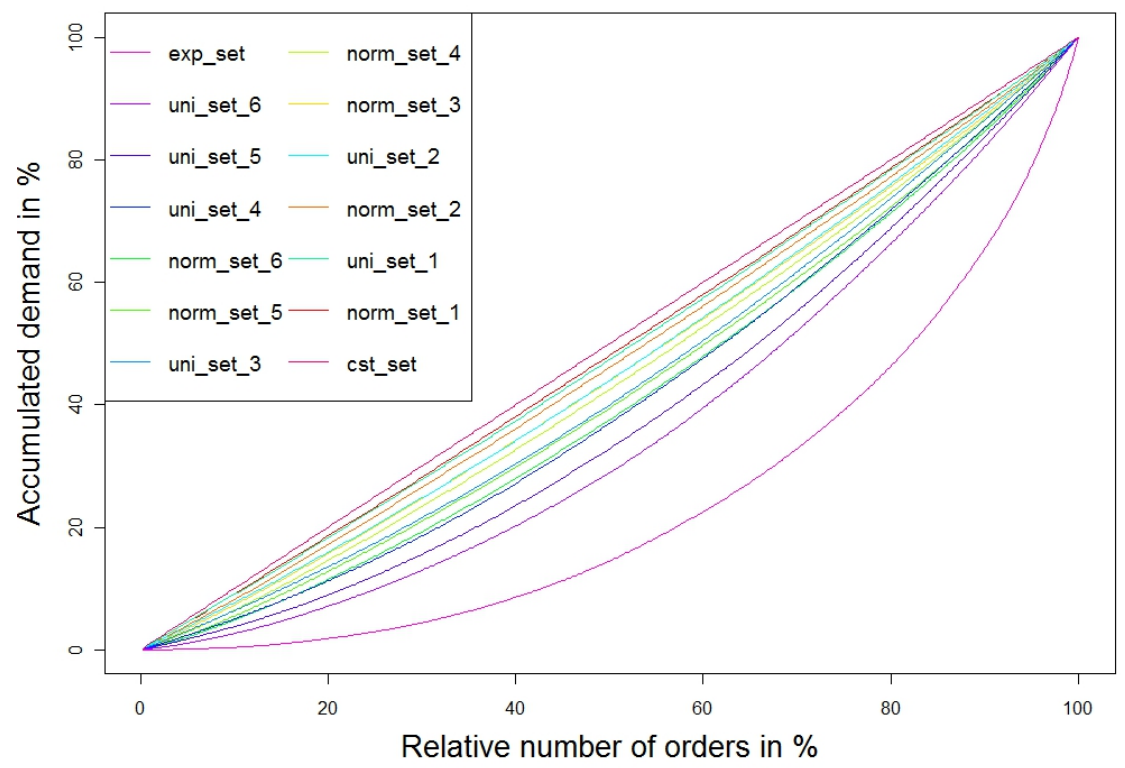

Fig. 2. The accumulation of demand graph for all 14 data sets used in the simulation study. Each line represents a different set of order size distributions. 
0.30

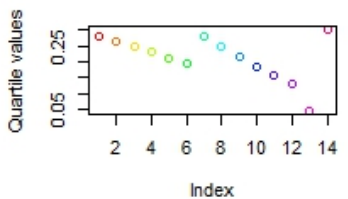

0.50

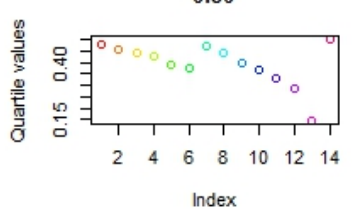

0.80

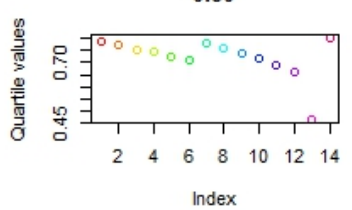

0.98

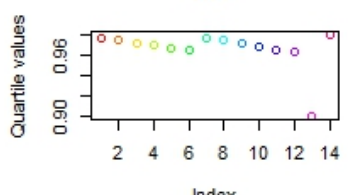

Index

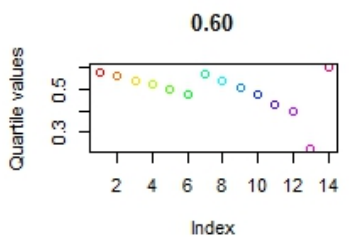

0.90

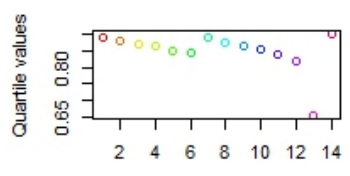

Index

0.99

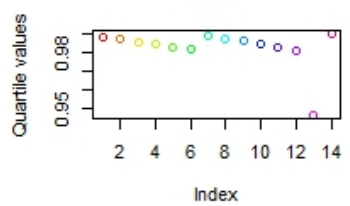

0.40

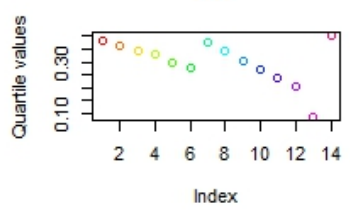

0.70

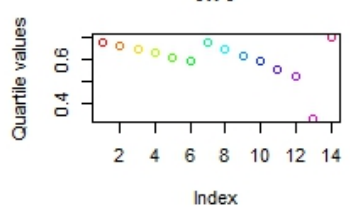

0.95

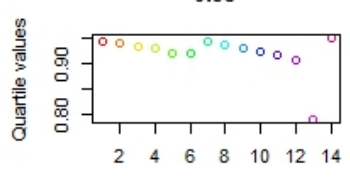

Index

Fig. 3. The quartiles from the 14 data sets used in the simulation study. Top left graph indicates color of each experiment.

Table 1. The descriptive statistics for both the order size distributions and the output stability of the manufacturing system per 1 hour

\begin{tabular}{|c|c|c|c|c|c|c|c|}
\cline { 2 - 8 } \multicolumn{1}{c|}{} & norm_set_1 & norm_set_2 & norm_set_3 & norm_set_4 & norm_set_5 & norm_set_6 & uni_set_1 \\
\hline $\mathrm{CV}_{\mathrm{Q}}$ & 0.05 & 0.10 & 0.15 & 0.19 & 0.27 & 0.31 & 0.06 \\
\hline $\mathrm{CV}_{\mathrm{V}, 1}$ & 0.12 & 0.16 & 0.17 & 0.21 & 0.21 & 0.24 & 0.13 \\
\hline $\mathrm{CV}_{\mathrm{N}, 1}$ & 0.12 & 0.16 & 0.17 & 0.20 & 0.21 & 0.25 & 0.13 \\
\hline & uni_set_2 & uni_set_3 & uni_set_4 & uni_set_5 & uni_set_6 & exp_set & cst_set \\
\hline $\mathrm{CV}_{\mathrm{Q}}$ & 0.14 & 0.23 & 0.31 & 0.40 & 0.48 & 1.04 & 0.00 \\
\hline $\mathrm{CV}_{\mathrm{V}, 1}$ & 0.14 & 0.20 & 0.23 & 0.26 & 0.33 & 0.53 & 0.10 \\
\hline $\mathrm{CV}_{\mathrm{N}, 1}$ & 0.15 & 0.20 & 0.21 & 0.26 & 0.31 & 0.67 & 0.10 \\
\hline
\end{tabular}

The processing and setup time should on average allow for 2 orders per interval (i.e. 2 orders per hour) and an output of 40 units per hour. As can be seen the output is quite naturally more unstable for the more unstable order size distributions. While $\mathrm{CV}_{\mathrm{N}, 1}$ and $\mathrm{CV}_{\mathrm{V}, 1}$ seem to be more or less perfectly correlated, there seems to be a 
marked difference when the exponentially distributed order sizes are considered. This is clearly seen from table 1 . This seems to indicate that the manufacturing system is more sensitive to asymmetrically distributed order sizes.

Table 2. Correlation values between order size volatility measures and output volatility

\section{Percentiles}

\begin{tabular}{|c|c|c|c|c|c|c|}
\hline & 0.30 & 0.40 & 0.50 & 0.60 & 0.70 & $\mathrm{CV}_{\mathbf{Q}}$ \\
\hline$C V_{\mathrm{N}, 1}$ & -0.972 & -0.980 & -0.987 & -0.991 & -0.993 & 0.992 \\
\hline \multirow[t]{2}{*}{$C V_{V, 1}$} & -0.927 & -0.943 & -0.957 & -0.972 & -0.985 & 0.987 \\
\hline & 0.80 & 0.90 & 0.95 & 0.98 & 0.99 & \\
\hline$C V_{N, 1}$ & -0.987 & -0.97 & -0.951 & -0.936 & -0.93 & \\
\hline $\mathrm{CV}_{\mathrm{V}, 1}$ & -0.995 & -0.997 & -0.991 & -0.984 & -0.981 & \\
\hline
\end{tabular}

As can be seen from table 2 strong correlation is found between all the order size stability measures. The strongest relations are for $\mathrm{CV}_{\mathrm{N}, 1}$ found in $\mathrm{CV}_{\mathrm{Q}}$ and the values for $50 \%-80 \%$ percentiles. This means that the $\mathrm{CV}_{\mathrm{N}, 1}$ is equally well described by the simple measure of $\mathrm{CV}_{\mathrm{Q}}$ and the percentiles. For $\mathrm{CV}_{\mathrm{V}, 1}$ the higher values (80\%-95\%) of the percentiles of order size distribution, better describe the variation of the output in form of total volume. So while $\mathrm{CV}_{\mathrm{Q}}$ is a an adequate predictor for the $\mathrm{CV}_{\mathrm{N}, 1}$ behavior, the order size volatility measures better describe the $\mathrm{CV}_{\mathrm{V}, 1}$.

Another interesting aspect that merits investigation is whether the output is in fact also independently distributed. To investigate whether the output is in fact independently distributed the autocorrelation function is used [2]. The conclusion is that both output measures are in fact highly dependent for almost all distributions. This could have big implications if e.g. the aim is to balance a production line [4], as dependently distributed output could indicate poor balance and difficulties in achieving a proper performance. Using Pearson correlation measures it is clear that there is a significant correlation (better than 0.01 ) between the input volatility and the lag 1 autocorrelation. This strongly indicates that while the input order sizes may independently distributed (as is the case for all but the constant order sizes), the volatility of order sizes actually has a strong impact on the output stability of the manufacturing system. So if a particular output rate is desired the volatility of order sizes must be taken into account. This is critical for any system reacting directly to customer orders as the dependencies of output directly influences the need to buffer as the need for buffering to achieve the same performance (e..g delivery time) increases as distributions go from independent to dependent. This can explain deviations in real life systems between expected and actual output, while having a relatively constant demand rate. Another observation is that the dependencies of output are quickly reduced after only a few periods (almost no lags greater than 1 are significant). This would seem to indicate that the disturbances tend to be absorbed by the system over time. The time horizon needed for the dependencies to disappear could be a valid measure for a reasonable delivery time for the system. 


\section{Conclusions and Further Research}

A number of significant conclusions can be reached based on the results presented in this paper. First, the new measures for order size volatility are robust estimators of volatility. Second, as order size volatility increases, the output volatility naturally increases also. Third, the need to aggregate in time to achieve a given stability of output is increasing disproportionally to the increases in order size volatility. Fourth, while the order sizes are i.i.d. the output is actually dependently distributed. The conclusion must by necessity be that the volatility of order sizes must be taken into account. Future research will focus on finding the aggregation of time (planning period length) needed to ensure that a given order size volatility can be used as input to a manufacturing system and give an output that can be considered stable.

\section{References}

1. Berry, W.L., Hill, T.: Linking Systems to Strategy. International Journal of Operations \& Production Management 12, 3-15 (1992)

2. Box, G.E.P., Jenkins, G.: Time Series Analysis: Forecasting and Control. Holden-Day (1976)

3. Brander, P., Levén, E., Segerstedt, A.: Lot sizes in a capacity constrained facility-a simulation study of stationary stochastic demand. International Journal of Production Economics 93-94, 375-386 (2005)

4. Nicholas, J.: Competitive Manufacturing Management: Continuous Improvement. Lean Production and Customer-focused Quality. McGraw-Hill, New York (1998)

5. Nielsen, P., Eriksen, T.: Towards an analysis methodology for identifying root causes of poor delivery performance. In: Conference Proceedings of IESM 2011, International Conference on Industrial Engineering and Systems Management (2011)

6. Nielsen, P., Nielsen, I., Steger-Jensen, K.: Analyzing and evaluating product demand interdependencies. Computers in Industry 61, 869-876 (2010)

7. Olhager, J.O.: Strategic positioning of the order penetration point. International Journal of Production Economics 85, 319-329 (2003)

8. Olhager, J., Wikner, J.: Master Production Scheduling. In: Beyond Manufacturing Resource Planning (MRPII): Advanced Models and Methods for Production Planning, pp. 3-20. Springer (1998)

9. R-project.org (webpage) (2012)

10. Silver, E.A., Pyke, D.F., Peterson, R.: Inventory Management and Production Planning and Scheduling, 3rd edn. John Wiley \& Sons (1998)

11. Vollmann, W., Berry, D., Whybark, T.E., Jacobs, F.: Manufacturing Planning and Control for Supply Chain Management. McGraw-Hill, Singapore (2005)

12. Wijngaard, J.: On Aggregation in Production Planning. Engineering Costs and Production Economics 6, 259-266 (1982)

13. Zotteri, G., Kalchschmidt, M., Caniato, F.: The impact of aggregation level on forecasting performance. International Journal of Production Economics 93-94, 479-491 (2005) 